\title{
Episodic memory contributions to autobiographical memory and open-ended problem-solving specificity in younger and older adults
}

\author{
Sarah L. Peters ${ }^{1} \cdot$ Carina L. Fan $^{1} \cdot$ Signy Sheldon $^{1}$ \\ Published online: 18 June 2019 \\ (C) The Psychonomic Society, Inc. 2019
}

\begin{abstract}
Research indicates that episodic memory processes are required to access specific autobiographical events and the details encompassed by a single event for several functions, including remembering and personal problem solving. Since healthy cognitive aging is associated with episodic memory decline, we hypothesized that older adults would be impaired at producing specific autobiographical events and details in service of these two functions. To test this hypothesis, younger and older adults completed two tasks (generation and elaboration) across two experiments (autobiographical memory and problem solving). The generation task required participants to produce multiple specific event memories or solutions to cues within a 90 -s time period. The elaboration task required participants to select a single memory or solution to describe in detail. We quantified the number of specific and non-specific responses provided during the generation task and scored the descriptions from the elaboration task for the number of episodic (internal) and non-episodic (external) details. Across experiment, older adults produced fewer specific responses (generation task) and fewer internal details (elaboration task) than younger adults. In addition, older adults generated more non-specific responses and external details than younger adults for the memory but not the problem-solving experiment. A correlational analysis showed that the number of specific responses (generation) and internal details (elaboration) correlated for the memory but not for the problem-solving experiment. These results show that age-related episodic memory decline impairs access to specific autobiographical events and detail information when remembering and problem solving, but that additional cognitive factors impact how these age declines present when solving problems.
\end{abstract}

Keywords Autobiographical memory $\cdot$ Episodic memory $\cdot$ Problem solving $\cdot$ Aging

\section{Introduction}

Episodic memory processes support the retrieval of specific autobiographical memories; however, autobiographical "specificity" can refer to the ability to access one autobiographical event but also retrieve the details embedded within a single autobiographical memory (Kyung, Yanes-Lukin, \& Roberts, 2016; Roberts, Yanes-Lukin, \& Kyung, 2018). Although models of autobiographical memory organization suggest that these forms of information are stored at different hierarchical levels (Burt, Kemp, \& Conway, 2003; Conway, 2005; Conway \& Pleydell-Pearce, 2000), episodic memory

Signy Sheldon

signy.sheldon@mcgill.ca

1 Department of Psychology, McGill University, 2001 McGill College Avenue, Montreal, Quebec H3A 1G1, Canada processes are required to access and associate both types of autobiographical knowledge in mind (Eichenbaum, Yonelinas, \& Ranganath, 2007; Naveh-Benjamin, Hussain, Guez, \& Bar-On, 2003; Olsen, Moses, Riggs, \& Ryan, 2012; Tulving, 2002). These same episodic memory processes are recruited in service of goal-directed cognitive tasks by helping to construct imagined or hypothetical events, which guide planning and problem solving (Addis, Wong, \& Schacter, 2008; Madore \& Schacter, 2014; Schacter et al., 2007; Sheldon, McAndrews, \& Moscovitch, 2011; Sheldon et al., 2015). Since episodic memory is a target of healthy cognitive aging (Dennis, Bowman, \& Peterson, 2014; McIntyre \& Craik, 1987; Spencer \& Raz, 1995), we tested how aging affects the ability to associate specific autobiographical events as well as the details of one event during autobiographical memory retrieval and when solving openended personal problems. Uncovering how age declines to episodic memory affect accessing different forms of specific 
autobiographical information for different purposes can improve our understanding of the organization and broader function of autobiographical knowledge.

\section{Autobiographical knowledge organization and retrieval}

According to the Self Memory System (SMS), autobiographical knowledge is stored in a hierarchy, meaning that autobiographical event information can be accessed as overarching themes (e.g., a lifetime period; "my teen years"), general events (the repeated aspects or concept of a memory; "going for dinner"), and critically as specific event memories (an event located in one time and place; "going for dinner with Claire last Sunday"; Conway, 2005; Conway \& PleydellPearce, 2000). According to this model, once a specific memory is accessed from this knowledge base, the precise details of the recalled event can be recovered from the lowest level of this hierarchy (e.g., contextual and perceptual details; "sitting in a dimly lit corner of the restaurant"). Another assumption of this model is that retrieving information from autobiographical knowledge, regardless of the level, will result in spreading activation to related elements (Conway, 2005). For instance, if a person retrieves a specific autobiographical memory in response to a general event cue ("going for dinner"), this will activate other specific memories related to that cue (e.g., other memories of going for dinner; Burt et al., 2003). In contrast, if a person retrieves specific details from a single autobiographical event, then there will be spreading activation to other event-specific details contained within that memory. There is a general consensus that episodic memory processes are needed to access specific event information, which includes both events and their related details, as well as to flexibly associate this information in mind (Eichenbaum et al., 2007; NavehBenjamin et al., 2003; Olsen et al., 2012; Tulving, 2002). As such, we hypothesize that episodic memory will be required both to access and to associate specific events and event details that are stimulated via spreading activation.

\section{Aging and autobiographical memory}

Healthy cognitive aging selectively affects the episodic memory component of autobiographical memory, leaving semantic processes relatively spared. As predicted by models of autobiographical knowledge organization, episodic memory deficits present as a decrease in the number of episodically derived or specific details used by older adults when describing past personal experiences (Devitt, Addis, \& Schacter, 2017; Levine, Svoboda, Hay, Winocur, \& Moscovitch, 2002; Madore, Gaesser, \& Schacter, 2014; Piolino, Desgranges, Benali, \& Eustache, 2002; Sheldon et al., 2011).

There is evidence that age-related episodic memory impairments extend beyond the level of detail, affecting the ability to access specific events. This leads older adults to rely on overgeneral (e.g., repeated or extended) event information, which is stored at a higher level in the autobiographical knowledge base than specific events (Bryan \& Luszcz, 2000; Holland \& Rabbitt, 1990; Parker \& Crawford, 1992; Piolino et al., 2006). Research testing younger and older adults on fluency measures that require participants to recall multiple specific event memories to a cue report that, within a fixed time-period, older adults generate fewer specific events than younger adults. Yet, there is debate as to whether this fluency deficit is due purely to episodic memory declines (i.e., difficulty accessing and associating together specific events) or is also influenced by deficits in executive processes supported by frontal lobe structures that are also affected in aging (Buckner, 2004; Cabeza \& Dennis, 2012; Piolino et al., 2010). Much like episodic decline affects the ability to associate the details of one memory, we contend that age-related episodic memory impairments contribute to deficits in associating multiple specific autobiographical events, above and beyond the contribution of agerelated executive decline (Dritschel, Williams, Baddeley, \& Nimmo-Smith, 1992; Martinelli et al., 2013; Piolino et al., 2010; Ros, Latorre, \& Serrano, 2009).

The above contention raises an important question about the relationship between autobiographical event specificity and detail. We propose that both forms of retrieval will require episodic memory processes to access specific information from the autobiographical knowledge base and then associate that information together. This fits with prior work indicating that common processes support retrieval when autobiographical information is accessed at the level of event or detail (Griffith et al., 2012; Ritchie, Skowronski, Richard Walker, \& Wood, 2006; Schacter, Addis, \& Buckner, 2007). In contrast, another body of research reports that accessing specific details versus events requires distinct cognitive processes, particularly those related to executive processing (Kyung, Yanes-Lukin, \& Roberts, 2016; Piolino et al., 2010; Roberts, Yanes-Lukin, \& Kyung, 2018). For instance, researchers have reported nonsignificant correlations between the ability to retrieve a specific memory and the amount of specific event detail that an individual feels they can recall (Kyung et al., 2016; Roberts et al., 2018). Thus, a secondary aim of our study is to clarify the link between event and detail specificity in autobiographical memory by exploring episodic process contributions to the retrieval of these two forms of autobiographical knowledge.

\section{Aging, autobiographical memory and open-ended problem solving}

It is now clear that the same episodic memory processes affected by aging also support several non-mnemonic tasks, including personal problem solving (Sheldon et al., 2011, Vandermorris, Sheldon, Winocur, \& Moscovitch, 2013; for related findings, see Jing, Madore, \& Schacter, 
2016; Madore \& Schacter, 2014). Common among these tasks is the need to form an autobiographical scenario to guide thinking and behavior, a capacity that is particularly critical for open-ended personal problems. Open-ended problems are those that do not have a clear solution or outcome (e.g., social problems such as the resolution of an interpersonal conflict). Following tenets laid out in the constructive episodic simulation hypothesis (Schacter \& Addis, 2009; Schacter et al., 2007), during open-ended problem solving, episodic memory processes support the flexible association of details from distinct autobiographical events to form a mental representation of a potential solution or hypothetical outcome. Previous work investigating the impact of healthy aging on open-ended problem solving has demonstrated that older adults use fewer episodic (i.e., specific) details and more general statements than younger adults when describing solutions, a finding that closely parallels those reported for autobiographical memory retrieval. Importantly, this age-related deficit in producing episodic details during problem solving has been linked to impairments in problem-solving efficacy, suggesting that episodic memory impairments have important functional consequences outside the domain of remembering (Madore \& Schacter, 2014; Sheldon et al., 2011; Vandermorris et al., 2013).

Whether age declines to episodic memory also affect generating specific solution options to personal problems is an open question. The problem-solving literature indicates that both episodic memory and executive control processes contribute to the production of solution options to open-ended problems (Channon \& Crawford, 1999; Kaiser et al., 2013). This may suggest that, much like for past events, older adults will be impaired at accessing multiple specific solutions. However, there is also evidence that older adults utilize different strategies than younger adults when generating solutions to personal problems (Mata, Schooler, \& Rieskamp, 2007; Sinnott, 1989). Older adults tend to take a more focused approach to interpersonal problem solving, relying on schema-like solutions that are based on lifetime experiential knowledge to think of what is "generally" done to solve a problem (e.g., to the problem "find new friends in a neighborhood," knowing that you "just join a club"). Younger adults tend to adopt a more exhaustive approach when generating solutions to a problem, possibly using episodic memory to simulate multiple novel outcomes to the problem (Berg, Meegan, \& Klaczynski, 1999; Blanchard-Fields, Mienaltowski, \& Seay, 2007; Channon \& Crawford, 2010; Crawford \& Channon, 2002). Although both strategies have been shown to lead to the generation of effective solutions, the approach utilized by older adults leads them to consider fewer solution alternatives than younger adults (Crawford \& Channon, 2002). What this suggests is that older adults may be recruiting distinct (non-episodic) processes during problem-solving to generate outcomes, meaning that, unlike autobiographical memory retrieval, the relationship between specificity when accessing and associating solutions versus the details of one simulated solution may diverge with age during open-ended problem solving.

\section{Current study}

From the literature described above, we set out to test three hypotheses:

1) Healthy cognitive aging affects the ability to access episodic memory details of one event (hereafter referred to as elaboration), both when retrieving autobiographical memories and when constructing detailed hypothetical solutions to open-ended personal problems.

2) Age-related deficits in episodic memory impairs the ability to associate together specific events (hereafter referred to as generation), when retrieving both autobiographical memories and constructed hypothetical solutions to openended problems.

3) Producing the specific details embedded within one event and generating specific events during autobiographical memory retrieval requires similar episodic memory processes to associate together autobiographical information, yet this relationship may change for problem solving if older adults strategically recruit alternate processes.

To test these hypotheses, we designed an experimental procedure in which we compared age effects to the generation and elaboration of autobiographical information for the functions of remembering and open-ended problem solving. Across two experiments, younger and older adults were presented with autobiographical memory or problemsolving cues. For each cue, they first accessed and associated together multiple specific memories or problem solutions (generation task). Then, they selected a single memory or solution to describe in detail (elaboration task). We used established scoring techniques to quantify the amount of specific episodic content for both tasks (Levine et al., 2002; Williams \& Broadbent, 1986). For the problemsolving experiment, we additionally scored the solution elaborations for problem-solving effectiveness, which has been linked to episodic memory functioning (Sheldon et al., 2011; Sheldon et al., 2015). Finally, we explored to relationship between event and detail specificity to assess if overlapping cognitive processes support specificity during the generation and elaboration tasks and if this relationship shifts with age. 


\section{Materials and method}

\section{Participants}

To reach a planned sample of 25 participants per age group, 34 healthy young adults were recruited through the McGill University Psychology participant pool and 28 healthy older adults were recruited from the local community. Sample sizes were based on prior related research (Addis, Musicaro, Pan, \& Schacter, 2010; Addis et al., 2008; Madore \& Schacter, 2014; Sheldon \& Chu, 2017; Sheldon et al., 2011; Vandermorris et al., 2013). As expected, some participants did not meet inclusion criteria, which required participants to be free of neurological or psychiatric disorders, speak fluent English, and have normal or corrected-to-normal hearing and vision. Of the younger adults, six were excluded from the analyses because they did not meet these criteria $(n=3)$, failed to return for the second testing session $(n=1)$, or did not follow testing instructions $(n=2)$. One young adult outlier was removed because the average length of their elaborations was greater than 2.5 standard deviations above the tested sample mean. This left a final sample of 27 young adults (seven male; age: $M=21.30$ years, $S E=0.49$; education: $M=15.30$ years, $S E=0.49$ ). Of the recruited older adults, four were excluded because they had indications of unhealthy cognitive aging based on a cognitive screening test (a score of lower than 24 out of 30 on the Montreal Cognitive Assessment ([MoCA]; Nasreddine et al., 2005). The Shipley Vocabulary subtest - a measure of crystallized intelligence - was also given to screen out older adult participants. All participants were above the suggested cut-off for healthy aging (a score of 33 out of 40; Mason \& Ganzler, 1964). One older adult participant was excluded because they did not return to complete the second experiment and two outliers were removed (due to lengthy elaborations, as above). This left a final sample of 22 older adults (eight male; age: $M=69.20$ years, $S E=1.05$; education: $M=16.40$ years, $S E=1.01$; MoCA score: $M=26.30, S E=$ 0.42 ; Shipley Vocabulary score: $M=35.90, S E=0.57$ ).

All participants provided written informed consent in accordance with the code of ethics established by the McGill University ethics review board. Participants were compensated for their time with either course credit (for students recruited from the psychology participant pool) or payment of Can $\$ 10$ per hour.

\section{Experimental overview}

Participants completed the autobiographical memory and problem-solving experiments in two separate 1-h sessions ( $M=4$ days apart). On average, more time elapsed between experiments for older adults ( $M=6.0$ days, range 1-21 days) than for younger adults $(M=2.2$ days, range $1-6$ days). This was driven by scheduling conflicts in three older adult participants who were tested 14,14 , and 21 days apart. When these three older adults were removed from the sample ( $M=4$ days, range 1-7 days), there was no appreciable difference between groups nor did removing these participants impact the pattern of results described below. Each experiment was presented via E-Prime experimental software (Version 2.0; Psychology Software Tools, Pittsburgh, PA, USA) and the order of experiments was counterbalanced across participants. An overview of the design, procedure, and measured outcome variables is presented in Fig. 1.

\section{Stimuli}

Sixteen cues (eight memory and eight problem solving; Table 1) were created based on previously published studies on autobiographical memory (Levine et al., 2002; Sheldon \& $\mathrm{Chu}, 2017$ ) and problem solving (Dritschel, Kogan, Burton, Esme, \& Goddard, 1998; Gerlach, Spreng, Madore, \& Schacter, 2014; Peters, Fellows, \& Sheldon, 2017; Platt \& Spivack, 1975). Half of the memory and problem cues were designed to be "high constraint," reflecting a task in which there is a strong cue-response association such that retrieved events are overlapping and highly similar to one another. The remaining half of the cues were designed to be "low constraint," reflecting a task in which cue-response associations are weak such that retrieved events are dissimilar from one another (Allaire \& Marsiske, 2002; Reed, Chan, \& Mikels, 2014). We refer the reader to the Supplementary Materials section of this paper for analyses and discussion of this cue manipulation, which is exploratory in nature and thus is not reported here.

\section{Procedure}

For each experiment (memory and problem solving), participants completed one practice trial with feedback, followed by eight randomly presented experimental trials in which they first completed the generation task, followed by the elaboration task.

Generation task Upon visual presentation of the cue, participants were given 90-s to think of as many memories or solutions as possible. For the memory experiment, participants were instructed to think of specific past personal events, those that occurred in a particular location and took place over minutes or hours but not longer than a single day. Participants were instructed to provide a short statement describing each event when it came to mind (e.g., "going to the park for my 10th birthday"). For the problem-solving experiment, participants were instructed to think of as many specific solutions an action or series of actions that could conceivably resolve the given problem - as possible and provide a short sentence describing that solution (e.g., "I would phone a friend for 


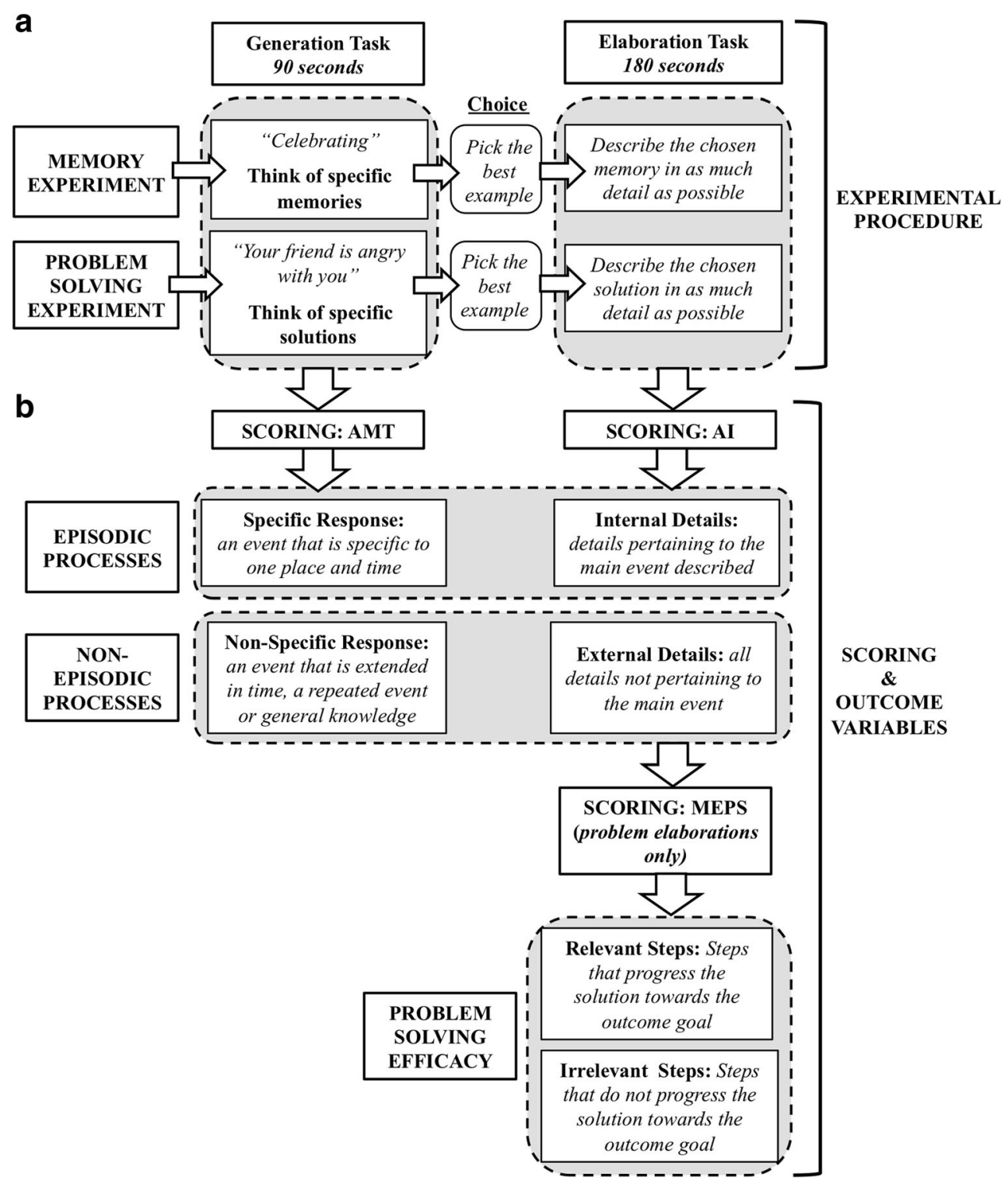

Fig. 1 Schematic representation of the experimental tasks, scoring procedure and outcome variables of interest. (A) The procedure for the autobiographical memory ("Memory") and the problem-solving experiments. Each experiment was subdivided into a generation and an elaboration task. (B) The scoring procedures and outcome variables for each task of the memory and problem-solving experiments. Adapted versions of the autobiographical memory test (AMT) and autobiographical interview (AI) scoring procedures were used to quantify episodic ("specific

help"). Throughout the generation task, two types of general prompts were given to participants. If a participant stopped responding, they were asked to "think of another memory (solution)." If a participant began elaborating (providing details) or generating a non-specific exemplar they were asked to "think of a more specific memory (solution)." In both cases, older adults were given significantly more prompts than younger adults $\left(t_{(46)}=6.29, p<.001\right)$ across both experiments. This type of general probing has been shown to have minimal impact on retrieval specificity in prior work (e.g., Levine et al., responses" and "internal details") and non-episodic ("non-specific responses" and "external details") content. Problem-solving elaborations were additionally scored with the standard method described in the Means End Problem Solving Test (MEPS) procedure, which distinguishes between and quantifies effective ("relevant steps") and ineffective ("irrelevant steps") problem solving in descriptions to open-ended problems

2002). All responses were recorded by the experimenter and shown to participants at the end of the 90-s generation period. Participants were instructed to select a single memory or solution that could be brought to mind clearly and were given as much time as needed to make this choice. Once a selection was made, the participants moved on to the elaboration task.

Elaboration task Participants were given 180-s (3 min) to describe the chosen memory or solution in as much detail as possible. These descriptions were audio recorded and later 
Table 1. Experimental cues used in the memory and problemsolving experiments

\begin{tabular}{ll}
\hline Memory cues & Problem cues \\
\hline Offices & You are running late and realize you have missed your plane \\
Galleries or venues & You want to go on a relaxing and affordable vacation \\
Home & You suspect you are going to be fired from your job \\
Travelling & You have recently moved into a new neighborhood and don't know anyone \\
Malls & You finish lunch at a restaurant and realize you have forgotten your wallet \\
Accomplishments & You want to plan a special day with your friends \\
Eating & You want to get a new pet that is not overly demanding \\
Celebrating & You suspect you are going to be fired from your job \\
\hline
\end{tabular}

transcribed for scoring. After each description, participants rated their memory and solution elaborations according to their experience of vividness $(0=$ not at all vivid to $100=$ extremely vivid) and familiarity with the scenario $(0=$ not at all familiar to $100=$ extremely familiar). Participants also provided a one-word description of the emotion associated with the elaborated memory or problem solution (e.g., "fear"), which was categorized as either positive or negative in emotional valence using the "Categorize Words" function of the Linguistic Inquiry and Word Count (LIWC) system (Pennebaker, Booth, \& Francis, 2007). For the memory experiment only, participants were asked to provide an estimate of the date of the event on a categorical scale ( $1=$ past week, 2 $=$ past year, $3=1$ to 5 years, $4=5$ to 10 years, $5=$ more than 10 years ago, $6=\mathrm{I}$ don't know).

\section{Scoring}

Generation task All generated responses were classified as specific or non-specific events or solutions using the standard procedure of the Autobiographical Memory Test (AMT; Williams \& Broadbent, 1986). For the memory experiment, responses were classified as specific if it was an event that occurred in a particular place and lasted less than a day (e.g., "my high school graduation ceremony") or non-specific if the response described an event extending beyond 1 day (e.g., "my trip to Paris"), a repeated event (e.g., "getting coffee every morning"), semantic information (e.g., "I have always been happy"), or was the repetition of a previously generated response. For the problem-solving experiment, solution responses were classified as specific if they described actions that could occur in one place and in less than a day (e.g., "I would look up information online") or non-specific if the response described an extended solution (e.g., "I would lose weight"), semantic information (e.g., "cats are low maintenance"), or was a repetition of a previous response. The average number of specific and non-specific responses across trials was used as the dependent factor in the reported analyses.

Elaboration task Descriptions were scored according to the procedure outlined in the Autobiographical Interview (AI) manual (Levine et al., 2002). Each description was segmented into details: distinct pieces of information (clauses) pertaining to an action, observation, thought, fact, or judgment. These details were classified as either internal or external. For the memory experiment, internal details were those pertaining directly to the main event described (e.g., who was there and perceptual, contextual, and emotional elements). A detail was classified as external if it related to an event outside the main event, or if it was factual, semantic, or metacognitive in nature. For the problem-solving experiment, details were scored according to an adapted version of the AI (e.g., Sheldon et al., 2011). Internal details were those relating directly to the narrative of the described solution path (e.g., actions taken, who was there). Semantic, off-topic, and metacognitive statements were scored as external. The elaborations for the problemsolving experiment were also scored using the standard method outlined in the Means End Problem Solving test (MEPS; Madore \& Schacter, 2014; Platt \& Spivack, 1975; Sheldon et al., 2011; Sheldon et al., 2015; Vandermorris et al., 2013). Solution descriptions were broken down into the number of steps described and these steps were categorized as relevant if they represented a logical progression from the problem state towards the solution (e.g., "I would pay the waitress") or irrelevant if it did not (e.g., "I would panic"; hopefully I will find my wallet"). The number of relevant steps is a standard measure of problem-solving effectiveness.

Scoring reliability Inter-rater reliability was assessed by calculating interclass correlation coefficients on a collection of randomly chosen descriptions for the three raters that scored the $\mathrm{AI}$ and AMT protocol and the two raters who scored the problem elaborations with the MEPS protocol. All reliability estimates were within acceptable limits $(r$ 's $>.80)$. All raters were blind to the age group and gender of the participant.

\section{Statistical analyses}

For the elaboration task subjective ratings, we ran mixed design ANOVAs on the average Likert-scale ratings (vividness, familiarity) or Chi-square tests of independence on ratings that were provided on categorical scales (emotional valence and 
memory date). For the elaboration task descriptions, we ran a mixed design ANOVA on the average number of details across trials, with age group (older adults, younger adults) as a between-subjects factor and experiment (memory, problem solving) and detail-type (internal, external) as within-subjects factors. A mixed design ANOVA was run on the average number of MEPS steps produced during the problemsolving experiment and included age group as a betweensubjects factor and step-type (relevant, irrelevant) as a within-subjects factor. For the generation task, we ran a mixed ANOVA on the average number of responses with age group as a between-subjects factor and experiment, and responsetype (specific, non-specific) as within-subjects factors. For all ANOVAs, significant effects were followed with Tukey's HSD post hoc contrasts. Finally, we ran a series of Pearson's correlational analyses between measures of specificity for the generation (specific responses) and elaboration tasks (internal details) across experiments for each age group.

\section{Results}

\section{Elaboration task}

Subjective ratings The mixed design ANOVA on the average vividness ratings with experiment as a between-subjects factor and age group (younger, older adults) as a within-subjects factor revealed a main effect of experiment $\left(F_{(1,47)}=17.01, p<.001\right.$, partial $\eta^{2}=.27$ ), which indicated that, overall, participants rated their memory elaborations $(M=82.6, S E=0.95)$ as more vivid than their problem-solving elaborations $(M=74.6, S E=1.26)$. We also found a main effect of age group $\left(F_{(1,46)}=5.32, p=\right.$ .043 , partial $\left.\eta^{2}=.12\right)$, where older adults $(M=81.6, S E=1.15)$ rated their elaborations as more vivid than younger adults $(M=$ $75.8, S E=1.11)$. There was no interaction between experiment and group $\left(F_{(1,47)}=1.53, p=.222\right.$, partial $\left.\eta^{2}=.03\right)$. The mixed design ANOVA on the average familiarity ratings with experiment and age group as factors showed no significant effects of experiment $\left(F_{(1,47)}=0.0001, p=.992\right.$, partial $\left.\eta^{2}=.00\right)$ or age group $\left(F_{(1,47)}=0.220, p=.641\right.$, partial $\left.\eta^{2}=.01\right)$, nor an interaction between experiment and age group $\left(F_{(1,47)}=1.16, p=\right.$ .287 , partial $\eta^{2}=.03$ ).

Chi-square tests of independence were used to examine ratings that were collected as categorical variables (emotional valence, memory date). For emotional valence categorization, the 2 (age group) $\times 2$ (emotional valence: positive, negative) contingency table indicated that the distribution of elaborations classified as positive or negative did not differ between younger and older adults for either the memory $\left(\chi_{(1)}^{2}=2.57, p=0.106\right.$, Cramer's $V=0.08)$ or the problem-solving $\left(\chi_{(1)}^{2}=2.91, p=\right.$ 0.088 , Cramer's $V=0.09)$ experiment. For date categorization, which was only collected for the memory experiment, the 2 (group) $\times 6$ (temporal interval: 1 - past week, 2 - past year, 3
-1 to 5 years, $4-5$ to 10 years, 5 - greater than 10 years, and 6 - don't know) contingency table indicated that memory elaborations were distributed differently across the date categories for older versus younger adults $\left(\chi_{(5)}^{2}=139.0, p<0.001\right.$, Cramer's $V=0.59$ ). Younger adults' elaborations were more likely to have occurred within the past 5 years (younger adults: $84.4 \%$ vs. older adults: $31.8 \%$ ) whereas older adults' elaborations were more likely to have occurred more than 5 years ago younger adults: $15.6 \%$ vs. older adults: $68.2 \%$ ).

Details A mixed design ANOVA on the average number of details produced with age group as a between-subjects factor and with experiment (memory, problem solving) and detail-type (internal, external) as within-subjects factors revealed main effects of experiment $\left(F_{(1,47)}=53.96, p<.001\right.$, partial $\left.\eta^{2}=0.53\right)$, detail-type $\left(F_{(1,47)}=239.08, p<.001\right.$, partial $\left.\eta^{2}=0.84\right)$, and age group $\left(F_{(1,47)}=4.43, p=.041\right.$, partial $\eta^{2}=0.09$ ). The effect of experiment was driven by more total details generated to memory $(M=36.7, S E=1.89)$ as compared to problem-solving cues $\left(M=25.0, S E=1.42 ; t_{(47)}=\right.$ $7.42, p<.001$, Cohen's $d=2.16$ ) and the effect of detail type was driven by the production of more internal $(M=22.6, S E=$ $0.46)$ as compared to external details $(M=9.32, S E=0.37$; $t_{(47)}=15.5, p<.001$, Cohen's $\left.d=4.54\right)$.

Focusing on age effects, younger adults $(M=33.50, S E=$ 1.77) generated more total details than older adults $(M=$ 27.50, $S E=2.31 ; t_{(47)}=2.19, p=.033$, Cohen's $\left.d=0.64\right)$. We also found a two-way interaction between detail-type and age group $\left(F_{(1,47)}=86.12, p<.001\right.$, partial $\left.\eta^{2}=0.13\right)$ and, importantly, a significant three-way interaction between experiment, detail-type, and age group $\left(F_{(1,47)}=8.42, p=\right.$ .006 , partial $\eta^{2}=0.15$; Fig. 2). The three-way interaction was explored with separate detail by age group ANOVAs for each experiment. For the memory experiment, younger adults $(M=31.60, S E=1.80)$ generated more internal details than older adults $\left(M=18.70, S E=1.82 ; t_{(47)}=4.98, p<.001, d=\right.$ $1.43)$, whereas older adults $(M=14.50, S E=0.77)$ generated

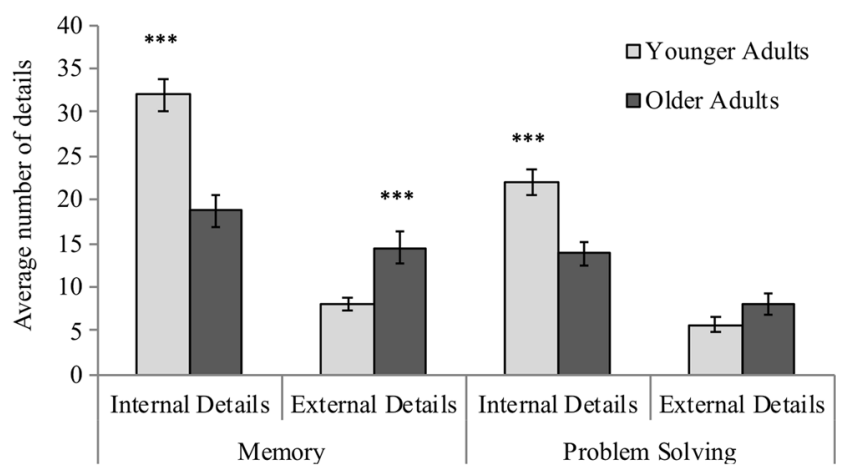

Fig. 2 Performance on the elaboration task for memory and problem solving. The average number of internal and external details generated by younger and older adults across the autobiographical memory and open-ended problem-solving experiments. Error bars represent standard error of the mean 
more external details than younger adults $(M=7.94, S E=$ $\left.0.75 ; t_{(47)}=-3.63, p<.001, d=-1.04\right)$. For the problemsolving experiment, younger adults $(M=21.90, S E=1.38)$ generated more internal details than older adults $(M=13.80$, $\left.S E=1.28 ; t_{(47)}=4.22, p<.001, d=1.21\right)$; however, older $(M$ $=7.99, S E=1.28)$ and younger adults $(M=5.69, S E=0.79)$ generated an equivalent number of external details $\left(t_{(47)}=-\right.$ $1.59, p=.118, d=-0.46)$.

As noted, for the memory experiment, older adults consistently selected more remote memories to elaborate upon relative to younger adults, which can affect detail generation (e.g., Piolino et al., 2002). To address the potentially confounding impact of retention interval, we repeated the above-reported mixed ANOVA with the average date of memory included as a covariate. The pattern of results emerging from this analysis was the same as that reported above (refer to Supplementary Materials for a complete report of this analysis).

Problem solving steps A mixed design ANOVA on the average number of generated steps with age group as a betweensubjects factor and with step-type (relevant, irrelevant) as a within-subject factor revealed main effects of step-type $\left(F_{(1,47)}\right.$ $=284.70, p<.001$, partial $\left.\eta^{2}=0.86\right)$ and age group $\left(F_{(1,47)}=\right.$ $11.80, p=.001$, partial $\left.\eta^{2}=0.20\right)$ and a significant age group $\times$ step-type interaction $\left(F_{(1,47)}=39.00, p<.001\right.$, partial $\eta^{2}=$ $0.45)$. The interaction effect was driven by younger adults $(M$ $=11.8, S E=0.57)$ describing more relevant steps than older adults $\left(M=6.98, S E=0.79 ; t_{(47)}=6.73, p<.001\right.$, Cohen's $d=$ 1.46). There was no difference between age groups in the number of irrelevant steps generated to problem cues $\left(t_{(93)}=-1.59\right.$, $p=.387$, Cohen's $d=0.03$; Fig. 3).

\section{Generation task}

Responses A mixed design ANOVA on the average number of responses generated with experiment (memory, problem

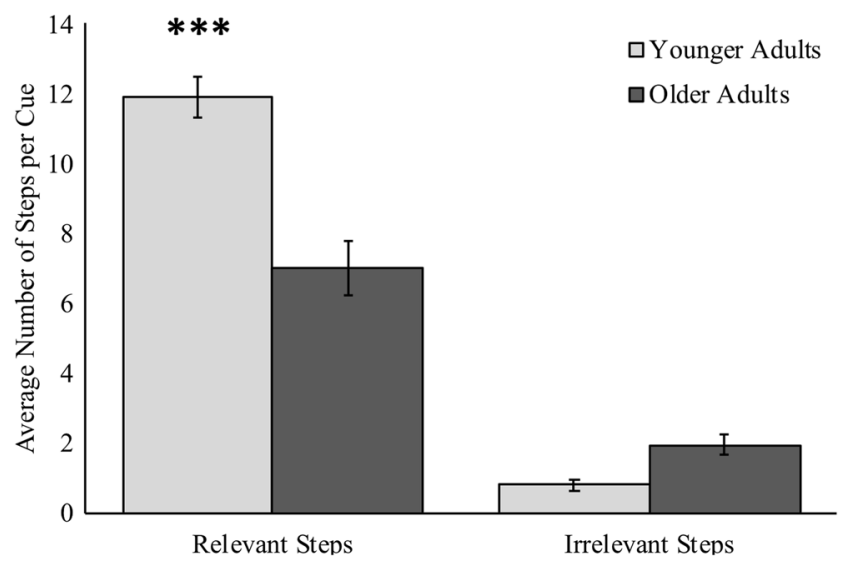

Fig. 3 The average number of steps (relevant versus irrelevant) generated for the problem-solving elaboration task by younger and older adults. Error bars represent standard error of the mean solving), response-type (specific, non-specific), and age group as factors revealed a main effect of response-type $\left(F_{(1,47)}=\right.$ 424.38, $p<.001$, partial $\left.\eta^{2}=0.90\right)$ and age group $\left(F_{(1,47)}=\right.$ $13.80, p<.001$, partial $\left.\eta^{2}=0.23\right)$ but not experiment $\left(F_{(1,47)}=\right.$ $0.93, p=.338$, partial $\left.\eta^{2}=0.02\right)$. More specific $(M=4.11, S E$ $=0.19)$ than non-specific $(M=1.67, S E=0.11)$ responses were generated, and younger adults $(M=6.53, S E=0.28)$ produced more responses than older adults $(M=4.96, S E=$ $0.33)$. We also found a significant response-type by age group interaction effect $\left(F_{(1,47)}=141.43, p<.001\right.$, partial $\left.\eta^{2}=0.75\right)$ and, importantly, a significant three-way interaction between response-type, experiment, and age group $\left(F_{(1,47)}=30.47, p<\right.$ .001 , partial $\eta^{2}=0.39$; Fig. 4). We investigated the latter threeway interaction with separate mixed design ANOVAs for each experiment, with response-type and age group as factors. For the memory experiment, younger adults $(M=5.10, S E=0.22)$ produced more specific exemplars than older adults $(M=$ $2.25, S E=0.19 ; t_{(47)}=9.21, p<.001$, Cohen's $\left.d=2.00\right)$, whereas older adults $(M=2.56, S E=0.19)$ produced more non-specific responses than younger adults $(M=1.44, S E=$ $0.19 ; t_{(47)}=-3.62, p=.003$, Cohen's $\left.d=0.79\right)$. For the problem-solving experiment, younger adults $(M=5.08, S E$ $=0.19)$ produced more specific responses than older adults $\left(M=3.68, S E=0.20 ; t_{(47)}=5.93, p<.001\right.$, Cohen's $d=$ $1.32)$; however, older adults $(M=1.47, S E=0.11)$ produced an equivalent number of non-specific responses to younger adults $\left(M=1.42, S E=0.15 ; t_{(47)}=-0.24, p=.995\right.$, Cohen's $d=0.05)$.

\section{The relationship between elaboration and generation}

A series of Pearson correlation coefficients were computed separately for younger and older adults between elaboration specificity (number of internal details) and generation specificity (number of specific responses) for the memory and problem-solving experiments (correlations are reported in

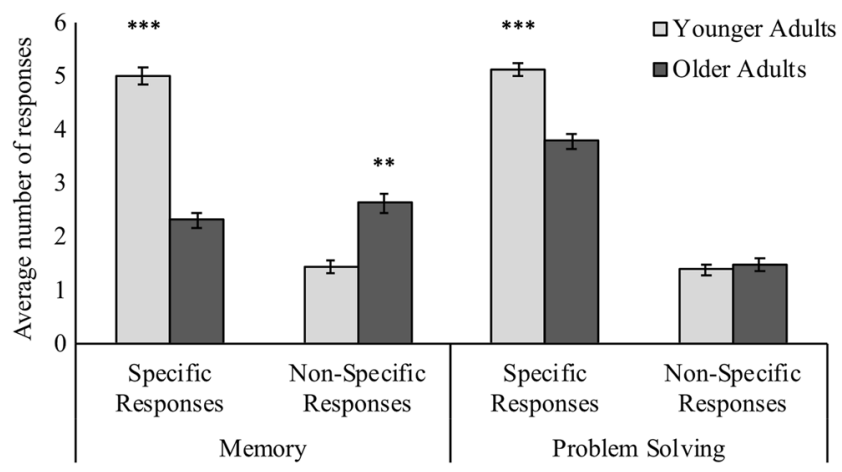

Fig. 4 Performance on the generation task for memory and problem solving. The average number of specific and non-specific exemplars generated by younger and older adults across the autobiographical-memory and problem-solving experiments. Error bars represent standard error of the mean 
Table 2). For the memory experiment, the number of specific responses during generation significantly correlated with the number of internal details produced during elaboration for both age groups. A Fisher r-to-z transformation indicated no difference between age groups in the strength of these correlations (Fisher-z $=-0.93, p=.17$ ). For the problem-solving experiment, the number of specific solutions produced during generation did not correlate with the number of internal details produced during elaboration in either age group. Across task, measures of generation specificity were marginally correlated for the younger adults, but strongly for the older adults. Likewise, measures of memory and problem-solving elaboration specificity were strongly correlated for older adults, but only weakly correlated for younger adults (Table 2).

As a secondary exploratory analysis, correlations between specificity scores for generation and elaboration were correlated with our measure of solution effectiveness (number of relevant steps; Table 2). We found a significant positive correlation between the number of relevant steps and problemsolving internal details across group, yet the strength of this relationship was significantly greater for older as compared to younger adults (Fisher- $z=-2.21, p=.013$ ). For younger adults, there was also a significant positive correlation between the number of relevant steps and specific solutions produced during generation, but not for the older adults.

\section{Discussion}

The primary goal of the present study was to understand how age-related changes in episodic memory affect the ability to generated multiple specific autobiographical events and to construct detailed representations of a single event in service of two functions: to recall the past and to solve open-ended personal problems. In response to autobiographical memory or problem-solving cues, younger and older adults completed two tasks, a "generation task," where they produced multiple events or solutions in a 90-s time frame, and an "elaboration task," where they selected a single memory or solution to describe in detail. We scored the generation task for the number of specific responses given and the elaboration task for the number of episodic (internal) details provided in the descriptions. These measurements were compared across the age groups and experiments (memory and problem solving), yielding the following results that relate to our three hypotheses: (1) We confirmed our first hypothesis that aging would selectively impair using episodic memory processes to construct a detailed event representation by showing an agedeficit in the ability to generate internal but not external details during the elaboration task across both experiments. (2) We confirmed our second hypothesis that age-deficits in episodic memory would extend to generating multiple specific autobiographical events with the finding that older adults produced fewer specific responses across both experiments. (3) We found partial support for our third hypothesis that generating specific events and details will similarly rely on episodic memory by showing that measures of specificity for the generation and elaboration task were correlated for the memory experiment but were unrelated for both younger and older adults for the problem-solving experiment. We discuss these findings in detail below.

\section{Aging and detailed autobiographical elaboration}

Results from the elaboration task showed that older adults were impaired at producing internal (episodic) but not external (non-episodic) details when describing autobiographical memories and problem-solving solutions. This finding replicates previous work demonstrating that age-related episodic deficits lead to complementary deficits in remembering and
Table 2 Correlation matrix for measures of specificity for the generation (average number of specific responses) and elaboration (average number of internal details) tasks across the autobiographical memory (AM) and problem-solving (PS) experiments for younger and older adults

\begin{tabular}{|c|c|c|c|c|c|}
\hline & $\begin{array}{l}\mathrm{AM} \\
\text { generation }\end{array}$ & $\begin{array}{l}\text { AM } \\
\text { elaboration }\end{array}$ & $\begin{array}{l}\text { PS } \\
\text { generation }\end{array}$ & $\begin{array}{l}\text { PS } \\
\text { elaboration }\end{array}$ & $\begin{array}{l}\text { PS } \\
\text { relevant steps }\end{array}$ \\
\hline \multicolumn{6}{|l|}{ Young adults } \\
\hline AM generation & - & & & & \\
\hline AM elaboration & $0.48 * *$ & - & & & \\
\hline PS generation & $0.40^{*}$ & 0.32 & - & & \\
\hline PS elaboration & 0.13 & 0.22 & 0.35 & - & \\
\hline PS relevant steps & 0.37 & 0.21 & $0.55 * * *$ & $0.57 * * *$ & - \\
\hline \multicolumn{6}{|l|}{ Older adults } \\
\hline AM generation & - & & & & \\
\hline AM elaboration & $0.67 * * *$ & - & & & \\
\hline PS generation & $0.54 * *$ & 0.34 & - & & \\
\hline PS elaboration & $0.54 * *$ & $0.66^{* * *}$ & 0.25 & - & \\
\hline PS relevant Steps & $0.60 * * *$ & $0.67 * * *$ & 0.29 & $0.87 * * *$ & - \\
\hline
\end{tabular}

Note. Pearson correlation coefficients; $* p<.05$. ** $p<.01$. *** $p<.001$ 
problem solving (Sheldon et al., 2011; Sheldon et al., 2015; Vandermorris et al., 2013) and, from a theoretical standpoint, supports the constructive episodic simulation hypothesis. This hypothesis suggests that episodic memory processes -that are affected by aging - are required to construct both past and hypothetical future events by accessing and associating together autobiographical details into a coherent mental representation (Schacter \& Addis, 2007; Schacter \& Addis, 2009; Szpunar, Addis, McLelland, \& Schacter, 2013).

Our study extends from prior work by providing new insights into how these representations are formed and affected in aging. For instance, we had participants choose which memory or solution to elaborate upon from a self-generated list of "options," which is unlike established studies in which participants were either restricted in their choice of scenario (e.g., first option generated) or one is provided to them. Laboratory-based studies have shown how the act of choosing alters (enhances) the use of episodic memory processes during decision-making tasks (Murty, DuBrow, \& Davachi, 2015), yet our data indicate that having the ability to choose a memory or problem solution for elaboration did not alleviate age-related deficits in detailed elaboration (also see Jing et al., 2016). In other words, older adults do not appear to counteract deficits in episodic memory during goal-directed tasks by self-selecting more "episodically accessible" events to describe from their autobiographical memory knowledge base, which has been reported in cases of amnesia (Lenton-Brym, Kurczek, Rosenbaum, \& Sheldon, 2016). We did find that older adults judged memory and solution elaborations as more vivid than younger adults. This could reflect a trend where older adults select different events for elaboration or simply that older adults assess vividness differently than younger adults. This latter point follows reports that older adults rely less on episodic content than younger adults to make vividness ratings (Comblain, D'Argembeau, \& Van der Linden, 2005; Johnson, Kuhl, Mitchell, Ankudowich, \& Durbin, 2015).

Similar to previous reports, we found that older adults described less effective solutions to the open-ended problems (i.e., fewer steps relevant to a solution were given in the elaboration task), which was correlated with their ability to generate specific episodic content when describing these solutions (a finding we return to below). We believe that this age deficit in providing episodically detailed and effective solutions relates to the type of problem cues used in the present study. Open-ended problems (e.g., planning a dinner party for new friends) lack a clear goal state or established solution path and are known to benefit from episodic memory processes to construct hypothetical outcomes and simulate solution paths (Allaire \& Marsiske, 2002; Madore et al., 2014; Madore \& Schacter, 2014; Pretz, Naples, \& Sternberg, 2003; Sheldon et al., 2011; Sheldon et al., 2015; Vandermorris et al., 2013). Whether these results would transfer to closed-ended problems would be an interesting research area to pursue (for some findings that speak to this point, see Vandermorris et al., 2013).

\section{Aging and generating specific autobiographical scenarios}

A more novel finding from our study was that older adults were impaired at generating multiple related specific autobiographical memories and solution options. Broadly, this finding suggests that older adults have difficulty accessing autobiographical information from the autobiographical knowledge base at the event level, which is known to rely on episodic memory to retrieve - or imagine - specific experiences (Conway, 2005). Given that we tested accessing specific event information with a fluency measure, our results indicate that older adults also have difficulty accessing related specific events that are made available via activation spreading to related nodes in the knowledge base (Conway, 2005). Since associating information in the mind depends on episodic memory processing (Eichenbaum, Yonelinas, \& Ranganath, 2007; Naveh-Benjamin, Hussain, Guez, \& Bar-On, 2003; Olsen, Moses, Riggs, \& Ryan, 2012; Tulving, 2002), we argue that age deficits during the generation task are partly due to episodic memory decline, which limits the ability to make use of the co-activated elements. Some empirical support for this argument comes from our study's correlational analyses, which showed that, for the autobiographical memory experiment, the number of internal details produced during the elaboration task - a confirmed metric of episodic memory use correlated with the number of specific responses provided during the generation task in both younger and older adults.

While we focus on episodic memory, others have explored how executive processes, also affected by age, impair fluently accessing specific autobiographical event information (Martinelli et al., 2013; Piolino et al., 2010). We do not dispute that there is a pivotal role of executive processes for both memory and problem solving, particularly in the generation task used here. Since our study did not include measures of executive functions, we are limited in speculating on their precise role; however, this would be a worthy avenue of future research. It may be that executive deficits account for the finding that older adults were largely less fluent than younger adults, generating fewer total details and responses (with the exception of total details for memory elaboration). Before expanding on this idea, we note that for the autobiographical memory experiment, older adults produced more nonepisodic information (external details, non-specific responses) than younger adults, which might reflect a shift from episodic to semantic processes as a strategy to access autobiographical information when associating both events and details (Devitt et al., 2017; Spreng et al., 2018). This was not found for the problem-solving experiment, which could be due to increased executive processing demands for this experiment that limited 
the ability of older adults to access "compensatory" nonspecific information. That is, the problem-solving experiment may have required more executive strategic search and information maintenance processes to generate and hold in mind novel solutions (Barbey \& Barsalou, 2009; D'Argembeau et al., 2010).

Speaking further to the problem-solving findings, prior work from aging research has suggested that older adults generate effective solutions to real-world personal problems, which has been attributed to a shift towards relying on accumulated semantic knowledge to support solution selection in older adults (Blanchard-Fields et al., 2007). This strategy, however, is not useful when multiple solutions must be generated that diverge from what is stored in semantic memory. In support, a previous report has shown that older adults can produce effective solutions to open-ended social problems, but generate fewer alternatives than younger adults (Channon \& Crawford, 1999). Thus, we argue that executive processes likely contribute to reduced fluency of solutions and memories - in older adults, but also that episodic memory processes affected by age limit the ability to produce multiple novel solutions via mentally simulating outcomes with autobiographical knowledge (Sheldon et al., 2015; Vandermorris et al., 2013). This argument is supported by the findings that older adults generated less effective solution paths, as measured by the number of relevant and logical steps described, when describing problem outcomes.

Finally, we noted a positive and relationship between measures of specificity during the elaboration and generation tasks for the autobiographical memory experiment, which appears to stand in contrast to Kyung et al. (2016), who failed to find a relationship between memory access and elaboration detail. However, there are some important methodological distinctions between our reported experiments and Kyung et al.'s (2016) work, particularly with respect to measures of specificity. In this prior report, detail specificity was measured as the number of non-redundant informational bits provided in brief, one- to two-sentence, written descriptions and event specificity as the proportion of specific memories generated across a series of cues. In our study, we measured detail by specifying the precise contribution of episodic memory processes to forming detailed representations, and our method for measuring generation specificity involved a fluency measure. Thus, it could be that different processes are recruited when retrieving one event versus multiple event details, but similar processes are recruited when associating together multiple instances of autobiographical knowledge at the level of event and detail (Eichenbaum et al., 2007; Naveh-Benjamin et al., 2003; Olsen et al., 2012; Tulving, 2002).

Interestingly, for both younger and older adults, we did not find a strong relationship between elaboration and generation specificity for the problem solving experiment. We speculate that problem-solving generation disproportionately relies on executive strategic search processes, whereas problem-solving elaboration, much like memory, requires episodic processes to simulate the solution in mind. Speaking further to problemsolving elaboration, we found a weak correlation between memory and problem-solving elaboration in younger adults but a strong and statistically significant correlation between these tasks for older adults. For the young adult cohort, one possibility alluded to by these results is that problem-solving elaboration recruits additional, non-episodic processes. Given the familiar nature of the presented problems, we propose that this task relies on semantic processes to access solution scripts and schemas, which facilitate solution simulation (Hershey, Walsh, Read, \& Chulef, 1990). Since older adults tend to approach cognitive tasks using semantic strategies, this could explain the stronger correlation between memory and problemsolving elaboration specificity in this cohort. Stated differently, these results suggest that older adults recruit non-episodic, likely semantic, processes similarly for both memory and problemsolving elaboration. These interpretations, while intriguing, are speculative and require further study.

More broadly, our correlational analyses showed that measures of specificity tended to be more strongly related in older adults as compared to younger adults. One possibility is that this pattern reflects a general bias towards recruitment of intact semantic processes in older adults, which could explain why measures of specificity were highly correlated across task and experiment. By contrast, younger adults may be able to flexibly recruit cognitive component processes (i.e., episodic, semantic, executive) as a function of task-specific demands, which could explain the relatively weaker correlations observed in this group. Alternatively, it is possible that older adults favor a distinct narrative style, which prioritizes the communication of personal meaning or the use of a more "explanatory" approach (Gaesser, Sacchetti, Addis, \& Schacter, 2011). This could influence or even partially explain the strong relationship between elaboration measures across experiment in older adults. Additional factors that may have commonly contributed to task performance in older adults include difficulty with task adherence due to age-related impairments in inhibiting irrelevant information or maintaining information in working memory (Piolino et al., 2010; Ros et al., 2009; Zacks \& Hasher, 1994) or a general age-related slowing in cognitive processing speed (Cerella \& Hale, 1994). Further research is required to confidently tease apart the relative contributions of these factors.

\section{Limitations and future directions}

The data we present here do not come without limitations and issues to consider. First, the modest sample sizes (young adult $n=27$, older adult $n=22$ ) may not have provided the appropriate power to detect some significant differences in our study, although it was in line with sample sizes reported in 
related work (Addis et al., 2010; Addis et al., 2008; Madore \& Schacter, 2014; Sheldon et al., 2011; Vandermorris et al., 2013). A second issue to consider is related to the memory and problem-solving cues. There was variability within the set of cues used in the present study, with some representing "high constraint" (i.e., well-defined scenarios) and some "low constraint" (i.e., more ambiguous or open-ended scenarios). Since this cue manipulation was relatively exploratory in nature, we did not include these results in our main analyses. We report this in our Supplementary Materials and believe that exploring this effect further could be a fruitful avenue of future research. Another avenue worth investigating is the precise episodic computations that contribute to the elaboration and generation tasks. Episodic memory encompasses both pattern completion and separation, which respectively involve using a cue to complete a memory or separating multiple instances in memory (Hunsaker \& Kesner, 2013; Yassa \& Stark, 2012). Although highly speculative, we propose that elaboration requires pattern-completion mechanisms to form a single mental representation and generation requires pattern-separation mechanisms to parse apart overlapping representations of similarly stored exemplars. There is empirical evidence that aging is accompanied by an imbalance between pattern separation and completion mechanisms (Yassa, Mattfeld, Stark, \& Stark, 2011; Yassa \& Stark, 2012). Future work may wish to test how established measures of behavioral pattern completion and separation (e.g., Stark, Yassa, Lacy, \& Stark, 2013) relate to performance on the presented experiments in aging. Finally, the current study did not include a comprehensive neuropsychological test battery, which limits our ability to comment confidently on the contribution of executive processes to our tasks and, more generally, to interpret correlation patterns in younger and older adults. It would be of great interest for future work to explore, in more detail, the relative contribution of cognitive component processes, particularly for the problem-solving experiment.

Even with these issues, the present study provides new data on the role of episodic memory processes in autobiographical memory and open-ended problem solving. We found that age deficits in using episodic memory for generating memory details extend to accessing specific information from a higher level of the autobiographical knowledge base hierarchy - that of the event - for both remembering and problem solving. These results highlight how episodic processes are commonly required to associate together specific autobiographical information for multiple functions, regardless of whether this is at the level of event or detail. By testing younger and older adults, our work also adds to a growing body of literature outlining the broad impact of episodic memory impairments in aging populations.

Acknowledgements We would like to thank Sonja Chu, Alexa Ruel, Julia Donahue, and Wendy Wang who transcribed or scored the memory and problem descriptions and Natasha Rajah and Lesley Fellows for providing access to healthy older adult participants. This research was supported by a grant from the Natural Sciences and Engineering Research Council (NSERC; RGPIN-04241) awarded to S. Sheldon.

\section{Compliance with ethical standards}

Open Practices Statement None of the data or materials for the experiments reported here are available via an online data repository but can be made available upon request to authors. None of the experiments reported herein were preregistered.

\section{References}

Addis, D. R., Musicaro, R., Pan, L., \& Schacter, D. L. (2010). Episodic simulation of past and future events in older adults: Evidence from an experimental recombination task. Psychology and aging, 25(2), 369-376.

Addis, D. R., Wong, A. T., \& Schacter, D. L. (2008). Age-related changes in the episodic simulation of future events. Psychological science, 19(1), 33-41.

Allaire, J. C., \& Marsiske, M. (2002). Well-and ill-defined measures of everyday cognition: relationship to older adults' intellectual ability and functional status. Psychology and aging, 17(1), 101-115.

Barbey, A., \& Barsalou, L. (2009). Reasoning and problem solving: models. Encyclopedia of neuroscience, 8, 35-43.

Berg, C. A., Meegan, S. P., \& Klaczynski, P. (1999). Age and experiential differences in strategy generation and information requests for solving everyday problems. International Journal of Behavioral Development, 23(3), 615-639.

Blanchard-Fields, F., Mienaltowski, A., \& Seay, R. B. (2007). Age differences in everyday problem-solving effectiveness: Older adults select more effective strategies for interpersonal problems. The Journals of Gerontology Series B: Psychological Sciences and Social Sciences, 62(1), P61-P64.

Bryan, J., \& Luszcz, M. A. (2000). Measurement of executive function: Considerations for detecting adult age differences. Journal of clinical and experimental neuropsychology, 22(1), 40-55.

Buckner, R. L. (2004). Memory and executive function in aging and AD: multiple factors that cause decline and reserve factors that compensate. Neuron, 44(1), 195-208.

Burt, C. D., Kemp, S., \& Conway, M. A. (2003). Themes, events, and episodes in autobiographical memory. Memory \& Cognition, 31(2), 317-325.

Cabeza, R., \& Dennis, N. A. (2012). Frontal lobes and aging. Principles of frontal lobe function. 2. New York: Oxford University Press, 628652.

Cerella, J., \& Hale, S. (1994). The rise and fall in information-processing rates over the life span. Acta psychologica, 86(2-3), 109-197.

Channon, S., \& Crawford, S. (1999). Problem-solving in real-life-type situations: The effects of anterior and posterior lesions on performance. Neuropsychologia, 37(7), 757-770.

Channon, S., \& Crawford, S. (2010). Mentalising and social problemsolving after brain injury. Neuropsychological Rehabilitation, 20(5), 739-759.

Comblain, C., D'Argembeau, A., \& Van der Linden, M. (2005). Phenomenal characteristics of autobiographical memories for emotional and neutral events in older and younger adults. Experimental aging research, 31(2), 173-189.

Conway, M. A. (2005). Memory and the self. Journal of Memory and Language, 53(4), 594-628. 
Conway, M. A., \& Pleydell-Pearce, C. W. (2000). The construction of autobiographical memories in the self-memory system. Psychological review, 107(2), 261-288.

Crawford, S., \& Channon, S. (2002). Dissociation between performance on abstract tests of executive function and problem solving in real-life-type situations in normal aging. Aging \& mental health, 6(1), 12-21.

D'Argembeau, A., Ortoleva, C., Jumentier, S., \& Van der Linden, M. (2010). Component processes underlying future thinking. Memory \& Cognition, 38(6), 809-819.

Dennis, N. A., Bowman, C. R., \& Peterson, K. M. (2014). Age-related differences in the neural correlates mediating false recollection. Neurobiology of Aging, 35(2), 395-407.

Devitt, A. L., Addis, D. R., \& Schacter, D. L. (2017). Episodic and semantic content of memory and imagination: A multilevel analysis. Memory \& Cognition, 1-17.

Dritschel, B., Kogan, L., Burton, A., Esme, B., \& Goddard, L. (1998). Everyday planning difficulties following traumatic brain injury: A role for autobiographical memory. Brain Injury, 12(10), 875-886.

Dritschel, B. H., Williams, J. M., Baddeley, A. D., \& Nimmo-Smith, I. (1992). Autobiographical fluency: A method for the study of personal memory. Memory \& Cognition, 20(2), 133-140.

Eichenbaum, H., Yonelinas, A. P., \& Ranganath, C. (2007). The medial temporal lobe and recognition memory. Annu. Rev. Neurosci., 30, 123-152.

Gaesser, B., Sacchetti, D. C., Addis, D. R., \& Schacter, D. L. (2011). Characterizing age-related changes in remembering the past and imagining the future. Psychology and aging, 26(1), 80-84.

Gerlach, K. D., Spreng, R. N., Madore, K. P., \& Schacter, D. L. (2014). Future planning: default network activity couples with frontoparietal control network and reward-processing regions during process and outcome simulations. Social cognitive and affective neuroscience, 9(12), 1941-1952.

Griffith, J. W., Sumner, J. A., Raes, F., Barnhofer, T., Debeer, E., \& Hermans, D. (2012). Current psychometric and methodological issues in the measurement of overgeneral autobiographical memory. Journal of behavior therapy and experimental psychiatry, 43, S21-S31.

Hershey, D. A., Walsh, D. A., Read, A. J., \& Chulef, A. S. (1990). The effets of expertise on financial problem solving: Evidence for goaldirected, problem-solving scripts. Organizational Behaviora and Human Decision Processes, 46(1), 77-101.

Holland, C., \& Rabbitt, P. (1990). Autobiographical and text recall in the elderly: An investigation of a processing resource deficit. The Quarterly Journal of Experimental Psychology, 42(3), 441-470.

Hunsaker, M. R., \& Kesner, R. P. (2013). The operation of pattern separation and pattern completion processes associated with different attributes or domains of memory. Neuroscience \& Biobehavioral Reviews, 37(1), 36-58.

Jing, H. G., Madore, K. P., \& Schacter, D. L. (2016). Worrying about the future: An episodic specificity induction impacts problem solving, reappraisal, and well-being. Journal of Experimental Psychology: General, 145(4), 402-418.

Johnson, M. K., Kuhl, B. A., Mitchell, K. J., Ankudowich, E., \& Durbin, K. A. (2015). Age-related differences in the neural basis of the subjective vividness of memories: Evidence from multivoxel pattern classification. Cognitive, Affective, \& Behavioral Neuroscience, 15(3), 644-661.

Kaiser, S., Simon, J. J., Kalis, A., Schweizer, S., Tobler, P. N., \& Mojzisch, A. (2013). The cognitive and neural basis of option generation and subsequent choice. Cognitive, Affective, \& Behavioral Neuroscience, 13(4), 814-829.

Kyung, Y., Yanes-Lukin, P., \& Roberts, J. E. (2016). Specificity and detail in autobiographical memory: Same or different constructs? Memory, 24(2), 272-284

Lenton-Brym, A., Kurczek, J., Rosenbaum, R. S., \& Sheldon, S. (2016). A new method for assessing the impact of medial temporal lobe amnesia on the characteristics of generated autobiographical events. Neuropsychologia, 85, 35-43.

Levine, B., Svoboda, E., Hay, J. F., Winocur, G., \& Moscovitch, M. (2002). Aging and autobiographical memory: dissociating episodic from semantic retrieval. Psychology and aging, 17(4), 677-689.

Madore, K. P., Gaesser, B., \& Schacter, D. L. (2014). Constructive episodic simulation: Dissociable effects of a specificity induction on remembering, imagining, and describing in young and older adults. Journal of Experimental Psychology: Learning, Memory, and Cognition, 40(3), 609-622.

Madore, K. P., \& Schacter, D. L. (2014). An episodic specificity induction enhances means-end problem solving in young and older adults. Psychology and aging, 29(4), 914-924.

Martinelli, P., Sperduti, M., Devauchelle, A.-D., Kalenzaga, S., Gallarda, T., Lion, S., ... Meder, J. F. (2013). Age-related changes in the functional network underlying specific and general autobiographical memory retrieval: a pivotal role for the anterior cingulate cortex. PloS one, 8(12), e82385.

Mason, C. F., \& Ganzler, H. (1964). Adult norms for the Shipley Institute of Living Scale and Hooper Visual Organization Test based on age and education. Journal of gerontology, 19(4), 419-424.

Mata, R., Schooler, L. J., \& Rieskamp, J. (2007). The aging decision maker: cognitive aging and the adaptive selection of decision strategies. Psychology and aging, 22(4), 796-810.

McIntyre, J. S., \& Craik, F. I. (1987). Age differences in memory for item and source information. Canadian Journal of Psychology/Revue canadienne de psychologie, 41(2), 175-192.

Murty, V. P., DuBrow, S., \& Davachi, L. (2015). The simple act of choosing influences declarative memory. Journal of Neuroscience, 35(16), 6255-6264.

Naveh-Benjamin, M., Hussain, Z., Guez, J., \& Bar-On, M. (2003). Adult age differences in episodic memory: further support for an associative-deficit hypothesis. Journal of Experimental Psychology: Learning, Memory, and Cognition, 29(5), 826.

Olsen, R., Moses, S., Riggs, L., \& Ryan, J. (2012). The hippocampus supports multiple cognitive processes through relational binding and comparison. Frontiers in human neuroscience, 6(146), 1-13.

Parker, D. M., \& Crawford, J. R. (1992). Assessment of frontal lobe dysfunction. A handbook of neuropsychological assessment, 267-291.

Pennebaker, J. W., Booth, R. J., \& Francis, M. E. (2007). Linguistic inquiry and word count: LIWC [Computer software]. Austin: liwc. net.

Peters, S. L., Fellows, L. K., \& Sheldon, S. (2017). The Ventromedial Frontal Lobe Contributes to Forming Effective Solutions to Real-world Problems. Journal of Cognitive Neuroscience, 29(6), 991-1001.

Piolino, P., Coste, C., Martinelli, P., Macé, A.-L., Quinette, P., GuilleryGirard, B., \& Belleville, S. (2010). Reduced specificity of autobiographical memory and aging: Do the executive and feature binding functions of working memory have a role? Neuropsychologia, 48(2), 429-440.

Piolino, P., Desgranges, B., Benali, K., \& Eustache, F. (2002). Episodic and semantic remote autobiographical memory in ageing. Memory 10(4), 239-257.

Piolino, P., Desgranges, B., Clarys, D., Guillery-Girard, B., Taconnat, L., Isingrini, M., \& Eustache, F. (2006). Autobiographical memory, autonoetic consciousness, and self-perspective in aging. Psychology and aging, 21(3), 510-525.

Platt, J. J., \& Spivack, G. (1975). Manual for the Mean-end Problem-solving Procedure (MEPS): A Measure of Interpersonal Cognitive Problemsolving Skill: Hahnemann Community Mental Health/Mental Retardation Center, Hahnemann Medical College and Hospital.

Pretz, J. E., Naples, A. J., \& Sternberg, R. J. (2003). Recognizing, Defining, and Representing Problems. In J. E. Davidson \& R. J. Sternberg (Eds.), The Psychology of Problem Solving: (pp. 3-30). Cambridge: Cambridge University Press. 
Reed, A. E., Chan, L., \& Mikels, J. A. (2014). Meta-analysis of the agerelated positivity effect: age differences in preferences for positive over negative information. Psychology and aging, 29(1), 1-15.

Ritchie, T. D., Skowronski, J. J., Richard Walker, W., \& Wood, S. E. (2006). Comparing two perceived characteristics of autobiographical memory: Memory detail and accessibility. Memory, 14(4), 471485.

Roberts, J. E., Yanes-Lukin, P., \& Kyung, Y. (2018). Distinctions between autobiographical memory specificity and detail: Trajectories across cue presentations. Consciousness and Cognition, 65, 342-351.

Ros, L., Latorre, J. M., \& Serrano, J. P. (2009). Working memory capacity and overgeneral autobiographical memory in young and older adults. Aging, Neuropsychology, and Cognition, 17(1), 89-107.

Schacter, D., \& Addis, D. (2007). The cognitive neuroscience of constructive memory: remembering the past and imagining the future. Philosophical Transactions of the Royal Society B: Biological Sciences, 362(1481), 773-786.

Schacter, D. L., \& Addis, D. R. (2009). On the nature of medial temporal lobe contributions to the constructive simulation of future events. Philosophical Transactions of the Roval Society of London B: Biological Sciences, 364(1521), 1245-1253.

Schacter, D. L., Addis, D. R., \& Buckner, R. L. (2007). Remembering the past to imagine the future: the prospective brain. Nature Reviews Neuroscience, 8(9), 657-661.

Sheldon, S., \& Chu, S. (2017). What versus where: Investigating how autobiographical memory retrieval differs when accessed with thematic versus spatial information. The Quarterly Journal of Experimental Psychology, 70(9), 1909-1921.

Sheldon, S., McAndrews, M., \& Moscovitch, M. (2011). Episodic memory processes mediated by the medial temporal lobes contribute to open-ended problem solving. Neuropsychologia, 49(9), 2439-2447.

Sheldon, S., Vandermorris, S., Al-Haj, M., Cohen, S., Winocur, G., \& Moscovitch, M. (2015). Ill-defined problem solving in amnestic mild cognitive impairment: Linking episodic memory to effective solution generation. Neuropsychologia, 68, 168-175.

Sinnott, J. D. (1989). A model for solution of ill-structured problems: Implications for everyday and abstract problem solving. In J. D. Sinnott (Ed.), Everyday problem solving: Theory and applications (pp. 72-99). New York: Praeger Publishers.
Spencer, W. D., \& Raz, N. (1995). Differential effects of aging on memory for content and context: a meta-analysis. Psychology and aging, 10(4), 527-539.

Spreng, R. N., Lockrow, A. W., DuPre, E., Setton, R., Spreng, K. A., \& Turner, G. R. (2018). Semanticized autobiographical memory and the default-executive coupling hypothesis of aging. Neuropsychologia, 110, 37-43.

Stark, S. M., Yassa, M. A., Lacy, J. W., \& Stark, C. E. L. (2013). A task to assess behavioral pattern separation (BPS) in humans: Data from healthy aging and mild cognitive impairment. Neuropsychologia, 51(12), 2442-2449.

Szpunar, K., Addis, D., McLelland, V., \& Schacter, D. (2013). Memories of the future: New insights into the adaptive value of episodic memory. Frontiers in behavioral neuroscience, 7(47), 1-3.

Tulving, E. (2002). Episodic memory: from mind to brain. Annual review of psychology, 53(1), 1-25.

Vandermorris, S., Sheldon, S., Winocur, G., \& Moscovitch, M. (2013). Differential contributions of executive and episodic memory functions to problem solving in younger and older adults. Journal of the International Neuropsychological Society, 19(10), 1087-1096.

Williams, J. M., \& Broadbent, K. (1986). Autobiographical memory in suicide attempters. Journal of abnormal psychology, 95(2), 144149.

Yassa, M. A., Mattfeld, A. T., Stark, S. M., \& Stark, C. E. L. (2011). Agerelated memory deficits linked to circuit-specific disruptions in the hippocampus. PNAS, 108(21), 8873-8878.

Yassa, M. A., \& Stark, C. E. L. (2012). Pattern seperation in the hippocampus. Trend in Neuroscience, 34(10), 515-525.

Zacks, R. T., \& Hasher, L. (1994). Directed ignoring: Inhibitory regulation of working memory. In D. Dagenbach \& T. H. Carr (Eds.), Inhibitory processes in attention, memory, and language (pp. 241264). San Diego: Academic Press.

Publisher's note Springer Nature remains neutral with regard to jurisdictional claims in published maps and institutional affiliations. 